\title{
Modality dependency of familiarity ratings of Japanese words
}

\author{
SHIGEAKI AMANO and TADAHISA KONDO \\ NTT Basic Research Laboratories, Atsugi, Kanagawa, Japan \\ and \\ KAZUHIKO KAKEHI \\ Nagoya University, Chikusa-Ku, Nagoya, Japan
}

\begin{abstract}
Familiarity ratings for a large number of aurally and visually presented Japanese words were measured for 11 subjects, in order to investigate the modality dependency of familiarity. The correlation coefficient between auditory and visual ratings was .808 , which is lower than that observed for English words, suggesting that a substantial portion of the mental lexicon is modality dependent. It was shown that the modality dependency is greater for low-familiarity words than it is for medium- or high-familiarity words. This difference between the low- and the medium- or high-familiarity words has a relationship to orthography. That is, the dependency is larger in words consisting only of kanji, which may have multiple pronunciations and usually represent meaning, than it is in words consisting only of hiragana or katakana, which have a single pronunciation and usually do not represent meaning. These results indicate that the idiosyncratic characteristics of Japanese orthography contribute to the modality dependency.
\end{abstract}

In addition to word frequency (Kucera \& Francis, 1967), word familiarity is an index of the relative ease of word perception. Although many studies show that a word's frequency affects its perception (e.g., Dupoux \& Mehler, 1990; Marslen-Wilson, 1990; Sègui, Mehler, Frauenfelder, \& Morton, 1982), some studies point out that familiarity is a better predictor than frequency for word perception (e.g., Gernsbacher, 1984; Gordon, 1985; Kreuz, 1987; Nusbaum, Pisoni, \& Davis, 1984).

Although these studies on familiarity dealt only with printed words (i.e., visually presented words), the findings may also be applicable to spoken words (i.e., aurally presented words), since some studies have shown that the effect of familiarity on word perception is not modality dependent. For example, Connine, Mullennix, Shernoff, and Yelen (1990) conducted naming experiments for auditory and visual stimuli and found that familiarity affected both types of stimuli in a similar fashion.

Pisoni and Garber (Garber \& Pisoni, 1991; Pisoni \& Garber, 1990) measured the familiarity ratings of 900 English words presented both aurally and visually. They found a high correlation between familiarity ratings using auditory and visual presentation (.93), and they suggested that the mental lexicon is amodal. Even if there are independent mental lexicons for auditory and visual words, they must be highly similar or closely connected.

A question that remains concerns whether the findings of Pisoni and Garber (Garber \& Pisoni, 1991; Pisoni \&

Correspondence should be addressed to S. Amano, NTT Basic Research Laboratories, 3-1 Morinosato Wakamiya, Atsugi-shi, Kanagawa Pref., 243-01, Japan (e-mail: amano@av-hp.ntt.jp).
Garber, 1990) are universal or language specific. That is, is the high correlation between auditory and visual familiarity ratings also observed in other languages? It is possible, for example, that the high correlation found by Pisoni and Garber is due to the relatively consistent relationship between English orthography and pronunciation. Such a relationship may result in a high correlation between auditory and visual familiarity ratings.

If this is correct, the correlation in languages with a more irregular relationship between orthography and pronunciation should be lower than that in English. For example, Japanese orthography is quite different from English orthography and is more complex. It uses three kinds of characters: kanji (based on Chinese ideograms), and two kinds of syllabaries, hiragana and katakana, both of which are essentially phonetic transcriptions. For example, the hiragana $\rightarrow$ and the katakana $力$ are always pronounced $/ \mathrm{ka} /$. Kanji, however, usually expresses meaning, and may have several different pronunciations; that is, kanji has no direct relationship to pronunciation. For example, the kanji 魔, which means evil, is always pronounced $/ \mathrm{ma} /$, whereas the kanji 下, which means down, is variously pronounced as $/ \mathrm{ka} /, / \mathrm{ge} /, / \mathrm{o} /, / \mathrm{sa} /$, /kuda/,/moto/,/simo/, and /sita/ (Nagasawa, Harada, \& Togawa, 1990). In the latter case (下), the pronunciation of kanji is decided according to the word in which the kanji is embedded. There are 83 hiraganas and 84 katakanas, whereas there are more than 10,000 kanjis. These three kinds of characters are used in combination, making the relationship between the Japanese orthography and pronunciation very complex.

It is also possible that the number of stimuli used in their familiarity-rating experiments might also serve to 
limit the generality of Pisoni and Garber's study (Garber \& Pisoni, 1991; Pisoni \& Garber, 1990): A total of 900 words may be too small to accurately represent a mental lexicon that must have a very large number of words. Although they selected words with various frequencies, too few stimuli might unexpectedly result in a high correlation between auditory and visual ratings.

To further investigate these questions, we used a large number of Japanese words and examined the correlation between familiarity ratings of words presented both aurally and visually.

\section{METHOD}

Two main experiments were conducted. Experiment 1 employed aurally presented words, and Experiment 2 employed visually presented words. Posttests were conducted in order to select those subjects whose ratings were reliable.

\section{Subjects}

Nineteen native speakers of Japanese (18-26 years old) participated in the experiments. They were paid for participating. All had graduated from high school, and had good reading skills and normal hearing ability.

\section{Materials}

A total of 62,001 words in the Shin-Meikai Japanese dictionary (Kindaichi, Kindaichi, Kenbo, Shibata, \& Yamada, 1974) were selected for use both in Experiment 1 and in Experiment 2. Two subsets of 3,100 words each were selected randomly from the original pool, one for an auditory posttest and the other for a visual posttest. No heterographic homophones (i.e., words having the same pronunciation as another word but a different orthography) or heterophonic homographs (i.e., words having the same orthography as another word but a different pronunciation) were included in these two posttest subsets, and the subsets shared no words in common.

A female native speaker of Japanese pronounced the 62,001 words at natural speaking rate. The stimuli for Experiment 1 were recorded digitally at a $16-\mathrm{kHz}$ sampling rate with $16-\mathrm{bit} \mathrm{A} / \mathrm{D}$ conversion. The stimuli were digitally stored in a personal computer (PC). Stimuli for Experiment 2 were prepared for presentation on a computer display using the same orthography as the Shin-Meikai Japanese dictionary: 40,794 stimuli were words consisting only of kanji, 2,985 stimuli were words consisting only of hiragana, and 4,654 stimuli were words consisting only of katakana. The remainder were combinations of kanji, hiragana, katakana, and a special character, such as a letter of an alphabet. The visual stimuli were presented using $32 \times 32$-pixel characters on a $640 \times 400$ pixel 15-in. computer display. They were viewed from a distance of about $0.5 \mathrm{~m}$.

\section{Procedure}

Experiment 1: Auditory presentation. The subjects were tested individually, using a PC. The auditory words were diotically presented at $75 \mathrm{~dB}$ (SPL) through headphones (STAX, SR- $\Lambda$ PRO). A different random order was used for each subject. The subjects were instructed to rate the familiarity of each word they heard. They made judgments by using a mouse to click a single digit on a seven-point scale (ranging from 1 for most unfamiliar to 7 for most familiar), which was displayed on the screen of the PC. The subjects were encouraged to use the full range of the scale. Each auditory word was successively presented approximately $1 \mathrm{sec}$ after the subject's rating of the previous word.

If the auditory word was a heterographic homophone, all alternative orthographies were visually presented on the display after the familiarity judgment had been made. The subjects were asked to select one of the orthographies, to identify which one had come to mind at the time of the initial familiarity rating. The subjects were then asked to rate the familiarity of the rest of the heterographic homophones successively.

For training trials, 200 stimuli from the original pool were randomly selected for each subject. The subjects participated in 200 training trials before participating in Experiment 1 . They performed approximately 2,000 judgments per day for six weeks.

Experiment 2: Visual presentation. As in Experiment 1, the subjects were individually tested, using a PC. They rated the familiarity of the visually presented words in the same manner as in Experiment 1.

If the visual word was a heterophonic homograph, all alternative pronunciations for the visual word were visually presented on the display in katakana after the familiarity judgment had been made. The subjects were asked to select one of the pronunciations, to identify which one had come to mind at the time of the initial familiarity rating. They were then asked to rate the familiarity of the remaining heterophonic homographs successively.

For training trials, 200 stimuli from the original pool were randomly selected for each subject. The subjects participated in 200 training trials before participating in Experiment 2. They performed approximately 3,000 judgments per day for four weeks.

Each subject participated in both Experiments 1 and 2. Ten subjects performed Experiment 1 first, and 9 subjects performed Experiment 2 first.

Posttests. All subjects participated in an auditory and a visual posttest $1-3$ months after the final experimental session. The procedures employed for the posttests were identical to those performed earlier.

\section{RESULTS AND DISCUSSION}

\section{Posttests}

Eleven of the 19 subjects ( 6 females and 5 males) were selected for the following analyses on the basis of their correlation coefficients for familiarity ratings between the posttest and the main experiment being higher than .5 both for aurally and visually presented words. For the 11 retained subjects, the mean correlation coefficient was $.703(\min =.536, \max =.854)$ for the aurally presented words, and $.738(\min =.570, \max =.851)$ for the visually presented words. Of the 11 retained subjects, 6 performed the auditory task first, and 5 performed the visual task first. The two tasks were still well counterbalanced across retained subjects.

\section{Main Experiments}

Frequency distributions of familiarity ratings for the 62,001 auditory and visual words are shown in Figure 1. The mean of visual familiarity ratings was $3.91(N=$ $62,001, S D=1.26$ ), and the mean of auditory familiarity ratings was $3.67(N=62,001, S D=1.27)$. These two mean values were significantly different $[t(62000)=$ $75.2, p<.0001]$.

To investigate the effects of modality on familiarity ratings, heterographic homophones and heterophonic homographs were excluded from the analyses. This was done because heterographic homophones were rated with their orthographies and heterophonic homographs were rated with their pronunciations represented in kata- 


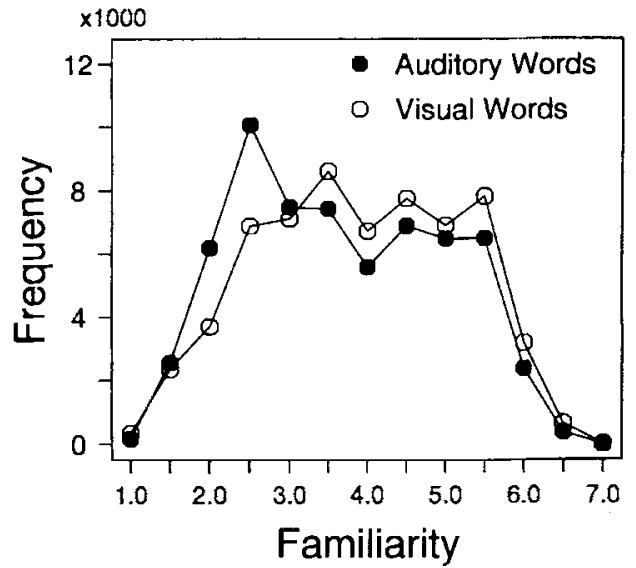

Figure 1. Frequency distributions of averaged familiarity ratings for 62,001 aurally and visually presented words.

kana in order to identify what the words were. This means that heterographic homophones were rated using dualmodality information, and that heterophonic homographs were rated using additional visual information; therefore the ratings of these words might be qualitatively different from those of single-solution words, which were rated using a single modality and with no additional information. ${ }^{1}$

After this exclusion from the total of 62,001 words, 31,702 single-solution words that had no heterographic homophones and no heterophonic homographs remained. Of these, 16,823 words consisted only of kanji, 2,144 words consisted only of hiragana, and 3,736 words consisted only of katakana.

For these 31,702 single-solution words, correlation coefficients of familiarity ratings between every pair of the selected 11 subjects were calculated. The mean of the correlation coefficients between subjects was as follows: $.549(N=55, \min =.409, \max =.736)$ for all the aurally presented words; $.559(N=55, \mathrm{~min}=.321$, $\max =.731$ ) for all the visually presented words; .556 $(N=55, \min =.343, \max =.719)$ for the visually presented words consisting only of kanji; $.549(N=55$, $\min =.188, \max =.774)$ for the visually presented words consisting only of hiragana; and $.627(N=55$, $\min =.463, \max =.800$ ) for the visually presented words consisting only of katakana. After averaging the familiarity ratings of each word over subjects, the mean of visual familiarity ratings was $3.92(N=31,702$, $S D=1.30$ ), and the mean of auditory familiarity ratings was $3.74(N=31,702, S D=1.31)$. These values were significantly different $[t(31701)=39.7, p<.0001]$.

The correlation coefficient between auditory and visual familiarity ratings was $.808(N=31,702)$. This is lower than the correlation coefficient of .93 reported by Pisoni and Garber for English words (Garber \& Pisoni, 1991; Pisoni \& Garber, 1990). This result shows that the familiarity ratings for Japanese words depend to a greater extent on the modality than do the ratings for English words.

As to what causes this lower correlation for Japanese words, it might be the characteristic Japanese orthography, which has many relationships to pronunciation. The use of kanji mainly contributes to this complex relationship, because most kanji characters have multiple pronunciations. On the other hand, hiragana and katakana have a one-to-one relationship to pronunciation, much like English orthography. Accordingly, it would be expected that the correlation coefficient for words consisting only of kanji would be lower than that for words consisting only of hiragana or katakana.

Such a difference was observed: The correlation coefficient between auditory and visual familiarity ratings for words consisting only of kanji was .767 ( $N=$ 16,823), which is much lower than that of $.924(N=$ $2,144)$ for words consisting only of hiragana $[|z|=26.3$, $p<.01$ ], and than that of $.950(N=3,736)$ for words consisting only of katakana $[|z|=45.3, p<.01]$. Interestingly, the correlation coefficients for words consisting only of hiragana or katakana are virtually the same as those found for English words (.93). The reason for the relatively high correlation in English therefore appears to be due to the fact that English orthography has more of a one-to-one relationship to pronunciation than does Japanese orthography. A similarly high correlation might be observed for other languages that have similar orthographic characteristics to English.

Pisoni and Garber (Garber \& Pisoni, 1991; Pisoni \& Garber, 1990) suggested that the mental lexicon is amodal, because the correlation between auditory and visual familiarity ratings was high. However, the present results indicate that such a modality independency is not necessarily true for languages other than English. The lower correlation for Japanese words suggests that a substantial portion of the mental lexicon is modality dependent for Japanese speakers, and that there may indeed be two independent mental lexicons, one auditory and one visual. These two systems must be similar, but they are apparently somewhat different.

What portion of the mental lexicon is modality dependent? More specifically, are low-familiarity words more modality dependent than medium- or high-familiarity words? To answer this question, the correlation between auditory and visual familiarity ratings was investigated for high-familiarity words, medium-familiarity words, and low-familiarity words.

There was a general tendency for the visual familiarity ratings to be higher than the auditory familiarity ratings. This difference between the auditory and visual familiarity ratings, although slight, was statistically significant, as described previously. For this reason, the familiarity ratings were transformed to $z$ scores. Highfamiliarity words were defined as those words with $z$ scores greater than 1.0. Medium-familiarity words were defined as those words with $z$ scores greater than 
Table 1

Correlation Coefficients ( $r$ ) Between Averaged Auditory Familiarity Ratings and Averaged Visual Familiarity Ratings as a Function of $z$-Score (All Words)

\begin{tabular}{|c|c|c|c|c|c|c|c|c|}
\hline \multirow{3}{*}{$\begin{array}{c}\text { Auditory } \\
\text { Familiarity } \\
\text { Rating }\end{array}$} & \multicolumn{8}{|c|}{ Visual Familiarity Rating } \\
\hline & \multicolumn{2}{|c|}{$z<-1$} & \multicolumn{2}{|c|}{$|z| \leq 1$} & \multicolumn{2}{|c|}{$1<z$} & \multicolumn{2}{|c|}{ All } \\
\hline & $r$ & $N$ & $r$ & $N$ & $r$ & $N$ & $r$ & $N$ \\
\hline$z<-1$ & .275 & 3,518 & .111 & 2,827 & .242 & 16 & .347 & 6,361 \\
\hline$|z| \leq 1$ & -.107 & 2,200 & .631 & 14,701 & .064 & 1,485 & .639 & 18,386 \\
\hline $1<z$ & .091 & 162 & -.104 & 1,897 & .543 & 4,896 & .330 & 6,955 \\
\hline All & .103 & 5,880 & .679 & 19,425 & .513 & 6,397 & .808 & 31,702 \\
\hline
\end{tabular}

Note- $N$, number of words contributing to the correlation.

-1.0 and less than 1.0. Low-familiarity words were defined as those words with $z$ scores that were less than -1.0 . Correlation coefficients between auditory and visual familiarity ratings as a function of $z$ scores are shown in Table 1.

Table 1 shows that the correlation coefficient of familiarity ratings between the low auditory $z$-score group and the low visual $z$-score group (.275) is much lower than that between the medium auditory $z$-score group and the medium visual $z$-score group $[.631 ;|z|=24.5$, $p<.01$ ] . It is also much lower than the correlation coefficient between the high auditory $z$-score group and the high visual $z$-score group $[.543 ;|z|=14.8, p<.01]$. This tendency was also observed in the correlation between the three visual $z$-score groups and the all auditory group, but was not observed in the correlation between the three auditory $z$-score groups and the all visual group. However, even in the latter case, the correlation coefficient between the low auditory $z$-score group and the all visual group (.347) was much lower than that between the medium auditory $z$-score group and the all visual group $[.639 ;|z|=27.1, p<.01]$.

These results indicate that low-familiarity words are more modality dependent than medium- or highfamiliarity words, and that the modality dependency of the mental lexicon is due to the influence of low-familiarity words.

What relationships are there between orthography and the modality dependency of low-familiarity words? To answer this question, correlation coefficients between the auditory and visual familiarity ratings for the three $z$-score groups were calculated for words consisting only of kanji (Table 2), for words consisting only of hiragana (Table 3), and for words consisting only of katakana (Table 4).

The correlation coefficient between the low auditory $z$ score group and the low visual $z$-score group for words consisting only of kanji $(.153)$ is much lower than that between the low auditory $z$-score group and the low visual $z$-score group for words consisting only of hiragana $[.571 ;|z|=8.42, p<.01]$, and is also much lower than that between the low auditory $z$-score group and the low visual $z$-score group for words consisting only of katakana $[.560 ;|z|=10.3, p<.01]$. In addition, comparisons among the $z$-score groups within words consisting only of kanji showed that the correlation coefficient between the low auditory $z$-score group and the low visual $z$-score group (.153) is much lower than that between the medium auditory $z$-score group and the medium visual $z$-score group $[.563 ;|z|=16.2, p<.01]$, and that it is also much lower than that between the high auditory $z$ score group and the high visual $z$-score group [.590; $|z|=15.5, p<.01]$. These findings show that kanji contributes to the low correlation between auditory and visual words in the low $z$-score group.

How, then, does kanji contribute to the overall correlation (.808) between aurally and visually presented words? The correlation coefficient between the high auditory $z$-score group and the high visual $z$-score group for words consisting only of kanji $(.590)$ is higher than that between the high auditory $z$-score group and the high visual $z$-score group for words consisting only of hiragana $[.385 ;|z|=3.80, p<.01]$ and for words consisting only

Table 2

Correlation Coefficients ( $r$ ) Between Averaged Auditory Familiarity Ratings and Averaged Visual Familiarity Ratings as a Function of $\boldsymbol{z}$-Score (Kanji-Only Words)

\begin{tabular}{|c|c|c|c|c|c|c|c|c|}
\hline \multirow{3}{*}{$\begin{array}{l}\text { Auditory } \\
\text { Familiarity } \\
\text { Rating } \\
\end{array}$} & \multicolumn{8}{|c|}{ Visual Familiarity Rating } \\
\hline & \multicolumn{2}{|c|}{$z<-1$} & \multicolumn{2}{|c|}{$|z| \leq 1$} & \multicolumn{2}{|c|}{$1<z$} & \multicolumn{2}{|c|}{ All } \\
\hline & $r$ & $N$ & $r$ & $N$ & $r$ & $N$ & $r$ & $N$ \\
\hline$z<-1$ & .153 & 1,313 & .136 & 1,511 & -.126 & 8 & .256 & 2,832 \\
\hline$|z| \leq 1$ & -.092 & 1,398 & .563 & 8,180 & .098 & 742 & .590 & 10,320 \\
\hline $1<z$ & .067 & 119 & -.115 & 945 & .590 & 2,607 & .344 & 3,671 \\
\hline All & -.005 & 2,830 & .599 & 10,636 & .562 & 3,357 & .767 & 16,823 \\
\hline
\end{tabular}

Note $-N$, number of words contributing to the correlation. 
Table 3

Correlation Coefficients ( $r$ ) Between Averaged Auditory Familiarity Ratings and Averaged Visual Familiarity Ratings as a Function of $z$-Score (Hiragana-Only Words)

\begin{tabular}{|c|c|c|c|c|c|c|c|c|}
\hline \multirow{3}{*}{$\begin{array}{c}\text { Auditory } \\
\text { Familiarity } \\
\text { Rating }\end{array}$} & \multicolumn{8}{|c|}{ Visual Familiarity Rating } \\
\hline & \multicolumn{2}{|c|}{$z<-1$} & \multicolumn{2}{|c|}{$|z| \leq 1$} & \multicolumn{2}{|c|}{$1<z$} & \multicolumn{2}{|c|}{ All } \\
\hline & $r$ & $N$ & $r$ & $N$ & $r$ & $N$ & $r$ & $N$ \\
\hline$z<-1$ & .571 & 375 & -.051 & 90 & & 1 & .563 & 466 \\
\hline$|z| \leq 1$ & .061 & 79 & .782 & 1,140 & .095 & 91 & .811 & 1,310 \\
\hline $1<z$ & & 0 & .213 & 153 & .385 & 215 & .437 & 368 \\
\hline All & .577 & 454 & .823 & 1,383 & .343 & 307 & .924 & 2,144 \\
\hline
\end{tabular}

Note- $N$, number of words contributing to the correlation.

of katakana $[.429 ;|z|=4.54, p<.01]$. However, the correlation coefficient between the medium auditory $z$-score group and the medium visual $z$-score group for words consisting only of kanji (.563) is lower than that between the medium auditory $z$-score group and the medium visual $z$-score group for words consisting only of hiragana $[.782 ;|z|=13.1, p<.01]$ and for words consisting only of katakana $[.855 ;|z|=25.0, p<.01]$. Therefore, in the high $z$-score group, kanji makes the overall correlation higher, but in the medium and low $z$-score groups, kanji makes it lower. However, kanji may make the overall correlation lower, because the summed number of words (i.e., 9,493$)$ in the low $z$-score group $(1,313)$ and medium $z$-score group $(8,180)$ is larger than the number of words in the high $z$-score group $(2,607)$. In addition, the kanji effects that lower the overall correlation can be more dominant than the effects of hiragana and katakana that heighten the overall correlation, because the number of words consisting only of kanji $(16,823)$ is much larger than the summed number of words consisting only of hiragana $(2,144)$ and katakana $(3,766)$ (i.e., 5,910). These factors result in the overall correlation between auditory and visual familiarity ratings being lower for Japanese than for English.

The present results suggest that kanji, which has oneto-many relationships between orthography and pronunciation, may contribute to the lower correlation of familiarity ratings between aurally and visually presented Japanese words. It is possible, however, that the lower correlation for Japanese words may be caused by other

Table 4

Correlation Coefficients ( $r$ ) Between Averaged Auditory Familiarity Ratings and Averaged Visual Familiarity Ratings as a Function of $z$-Score (Katakana-Only Words)

\begin{tabular}{|c|c|c|c|c|c|c|c|c|}
\hline \multirow{3}{*}{$\begin{array}{l}\text { Auditory } \\
\text { Familiarity } \\
\text { Rating } \\
\end{array}$} & \multicolumn{8}{|c|}{ Visual Familiarity Rating } \\
\hline & \multicolumn{2}{|c|}{$z<-1$} & \multicolumn{2}{|c|}{$|z| \leq 1$} & \multicolumn{2}{|c|}{$1<z$} & \multicolumn{2}{|c|}{ All } \\
\hline & $r$ & $N$ & $r$ & $N$ & $r$ & $N$ & $r$ & $N$ \\
\hline$<-1$ & .560 & 719 & .160 & 115 & & 0 & .558 & 83 \\
\hline$|z| \leq 1$ & -.021 & 110 & .855 & 1,892 & .137 & 181 & .869 & 2,183 \\
\hline $1<z$ & & 0 & .245 & 200 & .429 & 519 & .482 & 719 \\
\hline All & .547 & 829 & .873 & 2,207 & .456 & 700 & .950 & 3,736 \\
\hline
\end{tabular}

Note- $N$, number of words contributing to the correlation. characteristics of kanji. In particular, each kanji represents its meaning directly. This may result in a higher familiarity rating for the visually presented kanjicontaining words than for the corresponding aurally presented words. However, if the kanji is very unfamiliar and therefore not readable by the subjects, the visually presented kanji-containing words may have a lower familiarity rating than the corresponding aurally presented words. These characteristics of kanji may bidirectionally affect familiarity ratings, and hence may tend to lower the correlation between auditory and visual presentation. However, further investigation is necessary to confirm this hypothesis. In any case, it could be said that characteristics of Japanese orthography, particularly the special characteristics of kanji, affect word familiarity ratings, and that Pisoni and Garber's findings (Garber \& Pisoni, 1991; Pisoni \& Garber, 1990) cannot be directly applied to other languages that have different orthographic systems.

In summary, the present study compared familiarity ratings of aurally and visually presented Japanese words and showed that the correlation between auditory and visual familiarity ratings was lower than that observed for English words. It is suggested that the mental lexicon has some modality dependency, and that the idiosyncratic characteristics of Japanese orthography contribute to the modality dependency.

\section{REFERENCES}

Connine, C. M., Mullennix, J., Shernoff, E., \& Yelen, J. (1990). Word familiarity and frequency in visual and auditory word recognition. Journal of Experimental Psychology: Learning, Memory, \& Cognition, 16, 1084-1096.

Dupoux, E., \& MeHLER, J. (1990). Monitoring the lexicon with normal and compressed speech: Frequency effects and the prelexical code. Journal of Memory \& Language, 29, 316-335.

Garber, E. E., \& Prsoni, D. B. (1991). Lexical memory in visual and auditory modalities: A second report. Research on speech perception (Prog. Rep. No. 17, pp. 213-227). Bloomington: Indiana University, Speech Research Laboratory.

Gernsbacher, M. A. (1984). Resolving 20 years of inconsistent interactions between lexical familiarity and orthography, concreteness, and polysemy. Journal of Experimental Psychology: General, 113, 256-281.

Gordon, B. (1985). Subjective frequency and the lexical decision latency function: Implications for mechanisms of lexical access. Journal of Memory \& Language, 24, 631-645.

Kindaichi, K., Kindaichi, H., Kenbo, H., Shibata, T., \& Yamada, T. (Eds.) (1974)。亲明解国語辞典 (第 2 版) [Shin-Meikai Japanese dictionary (2nd ed.)]. Tokyo: Sansei-do.

Kreuz, R. J. (1987). The subjective familiarity of English homophones. Memory \& Cognition, 15, 154-168.

KuCERA, H., \& FRANCIS, W. N. (1967). Computational analysis of present-day American English. Providence, RI: Brown University Press.

Marslen-Wilson, W. D. (1990). Activation, competition, and frequency in lexical access. In G. T. M. Altmann (Ed.), Cognitive models of speech processing: Psycholinguistic and computational perspectives (pp. 148-172). Cambridge, MA: MIT Press.

NAGASAWA, K., HARADA, T., \& TOGAWA, Y. (Eds.) (1990). 新明解漢 和辞典 (第 4 版) [Shin-Meikai Chinese-Japanese dictionary (4th ed.)]. Tokyo: Sansei-do.

Nusbaum, H. C., Pisoni, D. B., \& Davis, C. K. (1984). Sizing up the Hoosier mental lexicon: Measuring the familiarity of 20,000 words. 
In Research on speech perception (Prog. Rep. No. 10, pp. 357-376). Bloomington: Indiana University, Speech Research Laboratory. PISONI, D. B., \& GARBER, E. E. (1990). Lexical memory in visual and auditory modalities: The case for a common mental lexicon. In Proceedings of the 1990 International Conference on Spoken Language Processing (pp. 401-404). Kobe, Japan: The Acoustical Society of Japan.

Segui, J., Mehler, J., Frauenfelder, U., \& Morton, J. (1982). The word frequency effect and lexical access. Neuropsychologica, 20, 615-627.

\section{NOTE}

1. This study was intended not only to investigate the modality dependency of familiarity but also to develop a familiarity database for aurally and visually presented Japanese words. It was therefore necessary to measure the familiarities of homophones and homographs, even if they were not used for analyzing the modality dependency.

(Manuscript received July 26, 1994;

revision accepted for publication December 29, 1994.) 